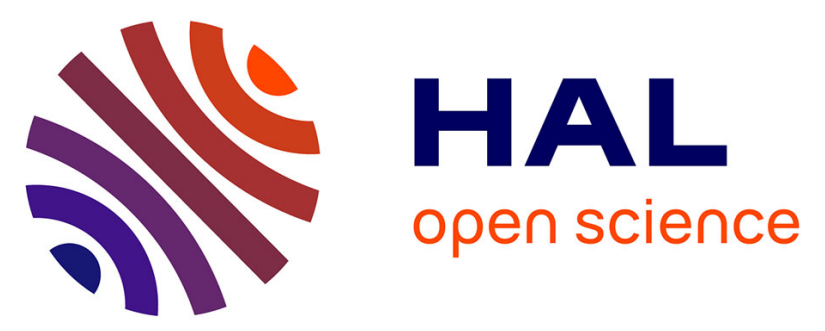

\title{
A Minimalist Approach for Identifying Affective States for Mobile Interaction Design
}

\author{
Subrata Tikadar, Sharath Kazipeta, Chandrakanth Ganji, Samit \\ Bhattacharya
}

\section{- To cite this version:}

Subrata Tikadar, Sharath Kazipeta, Chandrakanth Ganji, Samit Bhattacharya. A Minimalist Approach for Identifying Affective States for Mobile Interaction Design. 16th IFIP Conference on Human-Computer Interaction (INTERACT), Sep 2017, Bombay, India. pp.3-12, 10.1007/978-3319-67744-6_1. hal-01676156

\section{HAL Id: hal-01676156 \\ https://hal.inria.fr/hal-01676156}

Submitted on 5 Jan 2018

HAL is a multi-disciplinary open access archive for the deposit and dissemination of scientific research documents, whether they are published or not. The documents may come from teaching and research institutions in France or abroad, or from public or private research centers.
L'archive ouverte pluridisciplinaire HAL, est destinée au dépôt et à la diffusion de documents scientifiques de niveau recherche, publiés ou non, émanant des établissements d'enseignement et de recherche français ou étrangers, des laboratoires publics ou privés. 


\title{
A Minimalist Approach for Identifying Affective States for Mobile Interaction Design
}

\author{
Subrata Tikadar, Sharath Kazipeta, Chandrakanth Ganji and Samit Bhattacharya \\ Indian Institute of Technology Guwahati, India \\ subratatikadar@gmail.com, sharathkazipeta@gmail.com, \\ chandu8594@gmail.com, samit3k@gmail.com
}

\begin{abstract}
Human Computer Interaction (HCI) can be made more efficient if the interactive systems are able to respond to the users' emotional state. The foremost task for designing such systems is to recognize the users' emotional state during interaction. Most of the interactive systems, now a days, are being made touch enabled. In this work, we propose a model to recognize the emotional state of the users of touchscreen devices. We propose to compute the affective state of the users from 2D screen gesture using the number of touch events and pressure generated for each event as the only two features. No extra hardware setup is required for the computation. Machine learning technique was used for the classification. Four discriminative models, namely the Naïve Bayes, KNearest Neighbor (KNN), Decision Tree and Support Vector Machine (SVM) were explored, with SVM giving the highest accuracy of $96.75 \%$.
\end{abstract}

Keywords. Affective state, arousal and valence, emotion, touch gesture.

\section{Introduction}

With the ubiquitous explosion of mobile touch input devices, mobile HCI has become very important to improve usability of such devices. Affective touch interaction, which takes into account the affect and emotion of mobile touchscreen users, has the potential to significantly improve usability of these devices. The first and most important step for designing an affective interactive system is to recognize the affective state of the user. This may be followed by the design and implementation of appropriate interface and interaction that complement and/or change the users' emotional state. We can also make changes in the way tasks are performed depending on the current state of the user emotion. For instance, changing the look/contents of a webpage based on the emotional state of the user.

Mobile devices have limited capabilities in terms of their processor, storage and power back up. Moreover, the mobility aspect demands simplicity; these devices should not require too many extra attachments and wires. Literature contain many works on emotion recognition. In most of those cases, methods were proposed to recognize emotion from facial expression, gesture, posture and physiological signals. These mostly involved computer vision and image processing techniques, which are computationally expensive. In addition, such methods often require additional set ups such as cameras or probes and wires to record physiological signals. Consequently, 
most of the existing approaches are not suitable or feasible to detect emotional state of mobile users. The added hardware may affect the mobility and affordability of the devices. Therefore, an alternative approach is to predict affective state from the touch interaction behavior represented by the touch gestures (finger strokes). The finger strokes are indirect cues to the affective state of a touch screen user.

The indirect cue based approach has a major advantage: we do not require additional expensive sensors or wires to record the finger strokes. Further, the computations are much less compared to other approaches. We propose one such indirect cue based approach in this work.

A controlled experiment was conducted to establish the model with the empirical data of 29 users. For training and testing set of data, the random permutation cross validation technique' was used. Only two touch interaction characteristics: the number of touch events and average pressure of the events are used to determine the emotional state of a mobile touchscreen user in our proposed approach. We have not found any work that can detect emotion with such high accuracy from only two input features. Hence, our approach provides a unique and novel method. The proposed approach along with the data collection and analysis are described in this article.

\section{Related Works}

A wide class of emotion detection techniques use facial expressions (e.g., [12] [14] [18] [19] [25]), inspired by the theory proposed by Ekman et al [5]. Body movement and postures are also commonly used as cues to detect emotional states [3] [9]. Physiological signal such as electrooculogram (EOG), galvanic skin response (GSR), heart rate (HR), electrocardiogram (ECG), frontal brain electrical activity: electroencephalography (EEG) and eye blinking rate (EBR) were also used for emotion recognition (e.g., [1] [10] [16] [17] [18]). Some of the works are, in fact, based on the 'multimodal' approach. For instance, both EEG and peripheral physiological signal were used by Koelstra et al [17] for recognition of arousal-valence dimensional emotions induced by music videos.

All these works involve significant computations. Moreover, extra hardware, equipment and expensive sensors were used in most of those cases. We, on the other hand, are looking to detect the affective state of touch screen users, assuming small handled devices such as smartphones and tablets.

It has been reported that emotion can be recognized by keystroke patterns and dynamics [6] [15] [23] [35]. Our approach does not depend only upon keyboard dependent data. However, the works demonstrate that features such as pressure on a key or typing speed can be used to detect emotion.

Sano and Picard [30], Bauer and Lukowicz [2] and Ciman et al [4] used usersmartphone interaction data to detect the users' sate. The aim of these works was to identify the stress level of the users, so that it can be applied for taking care about the health condition of the users. We want to recognize more number of emotional states

\footnotetext{
${ }^{1} \mathrm{http} / /$ scikit-learn.org/stable/modules/cross_validation.html
} 
instead of restricting just two states like stress and no-stress. Moreover, some of these works [53] [2] used privacy sensitive data of the users like SMS, phone call, location etc. In our approach, we do not require such kind of personal or private data.

A system developed by Gao et al [8] was able to recognize the players' emotional states into four discrete states (Bored, Excited, Frustrated and Relaxed) as well as 2 levels of valence and 2 levels of arousal while playing game. They also used the finger stroke information for state prediction. However, emotion detection was done using the extracted touch information from a high-end device ( $\left.\mathrm{iPod}^{\mathrm{TM}}\right)$. Moreover, relatively large number of features (17 features) were used in their emotion recognition system. Shah et al [32] tried to overcome this limitation with a smaller set of features (10 features). However, some of these features, for instance, deviation in the number of strikes and deviation in the number of taps, may be difficult to know beforehand for use in automatic prediction. By considering just two features from the 2D screen (touch) gestures, number of touch events, and average pressure, our proposed approach addresses the issues discussed above. Consequently, the approach is expected to be less computational, easy to understand, and hence, easy to implement. The approach is described next.

\section{Proposed Approach}

In order to detect emotion, we propose to use discrete emotional states based on the circumplex model of affect [27] [28] [31]. An alternative set of circumplex emotional states, where the dimensional states are discretised, has been reported based on the arousal and valence level.

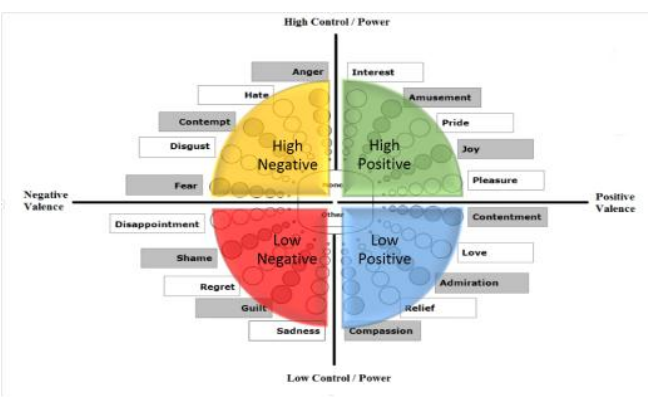

Fig. 1. The Geneva Emotional Wheel (GEW) and our proposed partitions of the wheel into four abstract discrete emotional states based on circumplex model of affect.

We based our idea of the four states on the Geneva Emotional Wheel (GEW ${ }^{2}$ ) [29] and the circumplex model of affect proposed by Posner et al [27]. The GEW is a theoretically derived and empirically tested instrument, to measure emotional reactions to objects, events, and situations. The GEW contains 20 different emotion families with their intensities diverging out as shown in Fig 1. The emotion families are arranged in

2 http://www.affective-sciences.org/en/gew/ 
a wheel shape with the axes being defined by two major dimensions of emotional experience: valence (x-direction) and arousal (y-direction).

We propose to represent each of the four quadrants of the GEW as a distinct emotional state based on the circumplex model of emotion. Thus, the top-right quadrant is the high positive state, the top-left quadrant is the high negative state, the bottom-left quadrant is the low negative state and the bottom-right quadrant is the low positive state. High and low indicate arousal level whereas positive and negative indicate valence level. The arousal-valence level emotional states are also equally important and are being identified and applied in many areas (e.g., [8] [21] [22] [33]). In fact, this type of states are more suitable in some particular research; e.g.; for identifying students' engagement level in classroom [34].

It has been reported that the user is likely to exert more pressure on the touch screen in high arousal state [8]. Number of touch events is also higher in the high arousal state [8] [35]. Typing speed also increases in high positive state [15]. The arousal dimension has been suggested to reflect the intensity of either appetitive or aversive systems [13] [20]. Aversive responses are high in high BIS (behaviour inhibition system) or BAS (behavioural activation system, formerly known as approach system). This clearly indicates that behavioural responses increase in high arousal state. Hence, the behavioural touch features such as frequency of touch, pressure applied while touching is expected to increase while a user is in high arousal state.

Depending on the value of number of events and pressure generated, it is possible to decide if the arousal level is high or low. The valence level is decided by assuming the basic human psychology that when a user is in positive mood s/he want to stay in that mood, but in case of negative mood s/he does not want to stay on that mood. When a player wins at the time of game playing, s/he doesn't want to lose. On the other hand, when s/he loses, s/he does not want to lose, s/he wants to win, i.e., s/he wants to change her/his state from negative to positive. This implies winning mode means positive valence, losing mood means negative valence. Previous works [7] [21] [24] [25] support this assumption. Once arousal levels are computed for every time interval, valence level can be measured by observing the rate of change of arousal.

We have used machine learning technique for the classification. Using the number of touch events and pressure generated on each event as feature attributes, our predictive model classifies the users into one of the four emotional states. We have conducted an empirical study with 29 users to build the classifier. The details of the empirical study are described in the next section.

\section{Details of Empirical Study}

\subsection{Experimental Setup}

In order to collect the emotional data of touchscreen users, we designed a small game application: 'Emotion Estimator' for Android OS. On the game screen, there are 4 buckets namely, 'BUCKET 1', 'BUCKET 2', 'BUCKET 3' and 'BUCKET 4'. At each instant, a ball is placed in one of the four buckets. The position of the ball changes once a player interacts with the touchscreen depending upon the modes de- 
scribed below. A player tries to guess in which bucket the ball is. Each wrong guess attracts a penalty of 2 and each correct guess gains an increment in score by 4 . Time is displayed at the top left corner of the screen and total score is displayed at the top right corner of the screen. The duration of the game is 2 minutes (120 seconds). Fig 2 shows the game interfaces for two particular instants.

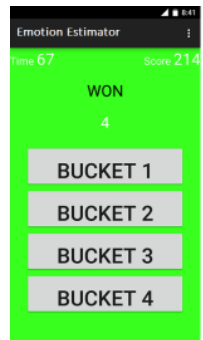

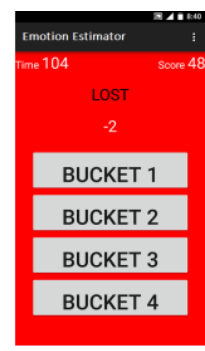

Fig. 2. The Emotion Estimator game interface with two possible situations. (a) is an example screenshot when a player guess right bucket, and hence, wins; (b) is an example screenshot when a player guess wrong bucket, and hence, loses.

The game consists of the following three modes. However, the players were not informed about these modes.

- Winning Mode: In this mode, the player always wins, irrespective of in which bucket the player tries to guess the ball is in.

- Losing Mode: In this mode, the player always loses, irrespective of in which bucket the player tries to guess the ball is in.

- Regular Mode: In this mode, the position of the ball changes after each touch interaction and if the player guesses correctly s/he wins, else s/he loses.

The game design strategy and timeline division is summarized in Fig 3.

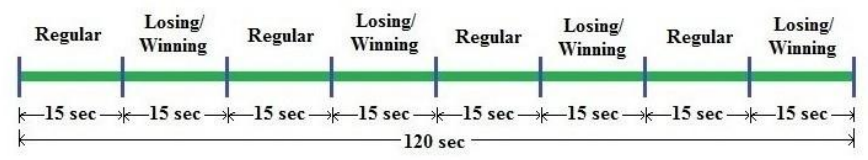

Fig. 3. Timeline division-strategy of the game for a particular instance.

We required 2 types of data: when a player wins \& when s/he loses. However, we placed a regular mode interval between a winning \& a losing mode interval so that within that interval the user's arousal level might be neutralized. It also helped not to overlap the 2 modes of the game. Random placing of winning and losing mode, each time the game started, has been followed to overcome the effect of biasness.

We chose $15 \mathrm{sec}$ interval for each mode because we assumed that 5 consecutive intervals (each of $3 \mathrm{sec}$ ) are sufficient to capture the emotional data of a user. We col- 
lected data of each mode for a particular user twice to analyze whether the nature of touch interaction data were same, for a particular user for a particular mode. At the same time, we couldn't choose longer interval for each mode because if a user won continuously for a long time, s/he might feel bored. Similarly, continuous losing might lead to user frustration.

In conventional way of data collection (asking the users about their emotional states), the quality of emotional data are not always good. The users themselves may not be sure about their emotional state. It may interrupt the interaction and may irritate the users also. So, we collected the data by the gaming strategy. Furthermore, the gaming approach is able to change the emotional state of the user naturally while playing the game. Generally, when a player wins, s/he feels positive (pleasure, joy, pride, admiration etc.). Similarly if a player lose, s/he feels negative (anger, disappointed, sadness, shame etc.). Hence, this helps us to gather the touchscreen data of different emotional state of the user.

The smartphone with which the data were collected was a 'OnePlus One' running on Cyanogen based OS 11 built on top of the Android Marshmallow. The screen size of the phone was 5.5 inch with resolution of 1080x1920 (401 pixels per inch density i.e. ppi). Number of touch events and pressure generated by each event were captured using the Android APIs onTouchEvent() and getPressure(), respectively.

\subsection{Participants}

We collected data from 29 users who voluntarily took part in the study. All of them were male UG/PG students of IIT Guwahati. The users were within the age group of 19-24 years, with the average age of 21 years. All of them were regular users of touchscreen devices, including both tablets and smartphones.

\section{Data Analysis}

From the empirical data of 29 users, we computed the sum of the number of touch events across the game playing modes for all the participants for each interval. Generally, a 'tap' is considered as 'Action Down' followed by 'Action Up' only, and occurs in an ideal case. 'Action Down' means putting the finger on touchscreen of the device. 'Action Up' means releasing the finger from the touchscreen. But in the real world, due to some movement of the finger while contacting with the touchscreen, there occurs an auxiliary action called 'Action Move' in between Action Down and Action Up, even in case of a tap. In this work all these three actions: 'Action Down', 'Action Move' and 'Action Up' are considered as events.

We assumed a $3 \mathrm{sec}$ time interval for data analysis. Choice of an appropriate time interval is not easy and we should keep in mind the way individuals react to stimuli. For example, emotional reaction to a text message may be dependent on a series of events and activities: getting a notification about the message, followed by reading the sender's name and figuring out the sender's identity (e.g., whether from boss or friend), followed by reading of the text. The affective state at the end of all these ac- 
tivities may depend on all of them. Thus, the particular state is arrived at after some seconds that was spent for all these activities. While trying to determine the affective state, we should consider this entire duration. We assume $3 \mathrm{sec}$ to be an appropriate duration to capture all the actions/events in any touch interaction in general and the game play scenario in particular that affects the emotional state of a user.

Along with the number of touch events, the average pressure of the touch events per time interval was also calculated. Equation (1) and equation (2) were used to calculate the number of events and the pressure of the touch events, respectively.

Let us consider that there are n number of taps that have taken place during the 3sec interval. For each of these taps, we determine the number of events $N i$. Number of events for a time interval is determined by calculating the sum of the number of events $\left(N_{i}\right)$ for $\mathrm{n}$ taps.

$$
\text { Number of events (in a particular interval) }=\sum_{i=1}^{n} N i
$$

For determining pressure value, let us consider there are $\mathrm{N}$ events in a time interval. Let $\mathrm{P}_{\mathrm{i}}$ be the pressure at each event. The average pressure is calculated as follows:

$$
\text { Average pressure }(\text { in a particular interval })=\left(\sum_{i=1}^{N} P i\right) / N
$$

\subsection{Classification of Data}

Four classifiers, namely the K-Nearest Neighbour (KNN), Support Vector Machine (SVM), Decision Tree and the Naïve Bayes have been explored. The observed accuracies were $94.57 \%, 96.75 \%, 96.4 \%$ and $88.4 \%$, respectively. Although the highest accuracy was found in case of SVM, the other classifiers also gave good enough results. Hence, we can claim that any of the four classifiers are equally applicable for our proposed model.

\section{Discussion}

We have found only one work (Gao et al [8]) which is similar to this work. They achieved $89 \%$ of accuracy while discriminating between 2 levels of arousal and 2 levels of valence. Whereas we have achieved highest accuracy of $96.75 \%$.

'Duration' along with pressure and frequency of touch could be considered while detecting the emotional state, but we avoided it because some of the previous works sometimes contradicted one another when duration was considered as a feature [8]. For instance, Matsuda et al [23] had shown that duration in the joy state is shorter and it is longer in the sad state. On the other hand, Hertenstein et al [11] observed that the touch duration in joy is longer than anger. Moreover, Use of only two features to detect the emotional state makes the model simpler and less expensive in terms of computing. 
We considered, as mentioned earlier, a 3 seconds interval for observing the rate of change of arousal. We considered this $3 \mathrm{sec}$ interval both in non-overlapping way ( 0 3, 3-6 ...) as well as in overlapping way $(0-3,1-4,2-5,3-6 \ldots)$. Considering the $3 \mathrm{sec}$ interval in overlapping way, number of data points increased to 1740 (29 users $\times 4$ winning/losing mode $\times 15$ interval for each mode) while that was 580 (29 users $\times 4$ winning/losing mode $\times 5$ interval for each mode) in case of non-overlapping way.

Although the data points varied, we could not find any variation in results. In both the cases, the prediction accuracy of four arousal-valence dimensional emotional state were same. This also indicate that the $3 \mathrm{sec}$ is a perfect choice of interval for measuring valence level in this context.

Other ways of expressing emotion, e.g., facial expression may be fake or disguised [15] [26]. Emotion detection by sensing physiological / psychophysiological signal, or even from multimodal approach may give better result [18] [19] [25], but these types of methods involve expensive hardware and equipment to be attached with the device. We have, therefore, chosen to consider the behavioral features to detect the emotional states. This fit best for the approach to detect emotional states of the users of the ubiquitously affordable touchscreen devices, with high accuracy.

We could not go for the questionnaire/ SAM (Self-Assessment Manikin) approach as we intended to follow the non-intrusive (i.e., without intruding the user) method to observe the change of the emotional state in real time. Therefore, an alternative approach to validate the model is to compare the emotional states calculated from physiological signal (like EEG recorded at the time of data collection) with the emotional states identified by our approach. We are currently working on this.

Naturally, someone may have doubt that something can be lost with this minimalist approach. Yes, as we're using only 2 features, it may not finely classify all the states, if the number of emotional states is large, e.g., 20. But for arousal-valence level emotion identification, our approach is good enough. In future, we plan to design more suitable game and to collect more data to refine and validate the model so that the identified emotional states can be used in real time affective systems, specifically in affective classroom.

\section{Conclusion}

A minimalist approach for identifying emotional state of a touchscreen user has been proposed in this work. We claim that only two features: number of touch events and pressure generated for each event are sufficient to identify the users' emotional state into any of the four states: high-positive, high-negative, low-positive and lownegative. High and low indicate the arousal level whereas positive and negative indicate the valence level. We intend to work on the use of affective state information for the interface and interaction design in future.

Acknowledgements. We are thankful to all the 29 users who voluntarily took part in the empirical studies. 


\section{References}

1. AlZoubi, Omar, Sidney K. D'Mello, and Rafael A. Calvo. "Detecting naturalistic expressions of nonbasic affect using physiological signals." IEEE Transactions on affective computing 3.3 (2012): 298-310.

2. Bauer, Gerald, and Paul Lukowicz. "Can smartphones detect stress-related changes in the behaviour of individuals?." Pervasive Computing and Communications Workshops (PERCOM Workshops), 2012 IEEE International Conference on. IEEE, 2012.

3. Camurri, Antonio, Ingrid Lagerlöf, and Gualtiero Volpe. "Recognizing emotion from dance movement: comparison of spectator recognition and automated techniques." International journal of human-computer studies 59.1 (2003): 213-225.

4. Ciman, Matteo, Katarzyna Wac, and Ombretta Gaggi. "iSenseStress: Assessing stress through human-smartphone interaction analysis." Pervasive Computing Technologies for Healthcare (PervasiveHealth), 2015 9th International Conference on. IEEE, 2015.

5. Ekman, Paul, E. Richard Sorenson, and Wallace V. Friesen. "Pan-cultural elements in facial displays of emotion." Science 164.3875 (1969): 86-88.

6. Epp, Clayton, Michael Lippold, and Regan L. Mandryk. "Identifying emotional states using keystroke dynamics." Proceedings of the SIGCHI Conference on Human Factors in Computing Systems. ACM, 2011.

7. Frederickx, Sofie, et al. "The relationship between arousal and the remembered duration of positive events." Applied Cognitive Psychology 27.4 (2013): 493-496.

8. Gao Yuan, Nadia Bianchi-Berthouze, and Hongying Meng. "What does touch tell us about emotions in touchscreen-based gameplay?." ACM Transactions on Computer-Human Interaction (TOCHI) 19.4 (2012): 31.

9. Glowinski, Donald, et al. "Toward a minimal representation of affective gestures." IEEE Transactions on Affective Computing 2.2 (2011): 106-118.

10. Hazlett, Richard L. "Measuring emotional valence during interactive experiences: boys at video game play." Proceedings of the SIGCHI conference on Human Factors in computing systems. ACM, 2006.

11. Hertenstein, Matthew J., et al. "The communication of emotion via touch." Emotion 9.4 (2009): 566.

12. Isbister, Katherine, et al. "The sensual evaluation instrument: developing an affective evaluation tool." Proceedings of the SIGCHI conference on Human Factors in computing systems. ACM, 2006.

13. Kambouropoulos, Nicolas, and Petra K. Staiger. "Personality and responses to appetitive and aversive stimuli: the joint influence of behavioural approach and behavioural inhibition systems." Personality and Individual Differences37.6 (2004): 1153-1165.

14. Kätsyri, Jari, and Mikko Sams. "The effect of dynamics on identifying basic emotions from synthetic and natural faces." International Journal of Human-Computer Studies 66.4 (2008): 233-242.

15. Khanna, Preeti, and M. Sasikumar. "Recognising emotions from keyboard stroke pattern." International journal of computer applications 11.9 (2010): 1-5.

16. Koelstra, Sander, et al. "Deap: A database for emotion analysis; using physiological signals." IEEE Transactions on Affective Computing 3.1 (2012): 18-31.

17. Koelstra, Sander, et al. "Single trial classification of EEG and peripheral physiological signals for recognition of emotions induced by music videos." Brain informatics. Springer Berlin Heidelberg, 2010. 89-100. 
18. Kolodyazhniy, Vitaliy, et al. "An affective computing approach to physiological emotion specificity: Toward subject-independent and stimulus-independent classification of film-induced emotions." Psychophysiology 48.7 (2011): 908-922.

19. Lang, Peter J., et al. "Looking at pictures: Affective, facial, visceral, and behavioral reactions." Psychophysiology 30.3 (1993): 261-273.

20. Lee, Seungjo, and Annie Lang. "Discrete emotion and motivation: Relative activation in the appetitive and aversive motivational systems as a function of anger, sadness, fear, and joy during televised information campaigns."Media Psychology 12.2 (2009): 148-170.

21. Lerner, Jennifer S., and Dacher Keltner. "Beyond valence: Toward a model of emotionspecific influences on judgement and choice." Cognition \& Emotion 14.4 (2000): 473-493.

22. Lottridge, Danielle, Mark Chignell, and Aleksandra Jovicic. "Affective interaction understanding, evaluating, and designing for human emotion."Reviews of Human Factors and Ergonomics 7.1 (2011): 197-217.

23. Matsuda, Yasuhiro, et al. "Emotional communication in finger braille." Advances in $\mathrm{Hu}-$ man-Computer Interaction 2010 (2010): 4.

24. Murphy, Sheila T., and Robert B. Zajonc. "Affect, cognition, and awareness: affective priming with optimal and suboptimal stimulus exposures." Journal of personality and social psychology 64.5 (1993): 723.

25. Partala, Timo, and Veikko Surakka. "The effects of affective interventions in humancomputer interaction." Interacting with computers 16.2 (2004): 295-309.

26. Picard, Rosalind W. "Affective computing: challenges." International Journal of HumanComputer Studies 59.1 (2003): 55-64.

27. Posner, Jonathan, James A. Russell, and Bradley S. Peterson. "The circumplex model of affect: An integrative approach to affective neuroscience, cognitive development, and psychopathology." Development and psychopathology 17.03 (2005): 715-734.

28. Russell, J. A. A Circumplex Model of Affect. Journal of Personality and Social Psychology,39(6), 1161-1178, 1980.

29. Sacharin, Vera, Katja Schlegel, and K. R. Scherer. "Geneva emotion wheel rating study." Center for Person, Kommunikation, Aalborg University, NCCR Affective Sciences. Aalborg University, Aalborg (2012).

30. Sano, Akane, and Rosalind W. Picard. "Stress recognition using wearable sensors and mobile phones." Affective Computing and Intelligent Interaction (ACII), 2013 Humaine Association Conference on. IEEE, 2013.

31. Schlosberg, Harold. "Three dimensions of emotion." Psychological review 61.2 (1954): 81.

32. Shah, Sachin, J. Narasimha Teja, and Samit Bhattacharya. "Towards affective touch interaction: predicting mobile user emotion from finger strokes." Journal of Interaction Science 3.1 (2015): 1-15.

33. Stickel, Christian, et al. "Emotion detection: application of the valence arousal space for rapid biological usability testing to enhance universal access." International Conference on Universal Access in Human-Computer Interaction. Springer Berlin Heidelberg, 2009.

34. Woolf, B., Burleson, W., Arroyo, I., Dragon, T., Cooper, D. and Picard, R. (2009) 'Affect aware tutors: recognising and responding to student affect', Int. J. Learning Technology, Vol. 4, Nos. 3/4, pp.129-164.

35. Zimmermann, Philippe, et al. "Affective computing — a rationale for measuring mood with mouse and keyboard." International journal of occupational safety and ergonomics 9.4 (2003): 539-551. 